\title{
When Do Essential Tremor Patients Develop Head Tremor? Influences of Age and Duration and Evidence of a Biological Clock
}

\author{
Elan D. Louis ${ }^{\mathrm{a}-\mathrm{d}}$ \\ ${ }^{a}$ G.H. Sergievsky Center, ${ }^{b}$ Department of Neurology and 'Taub Institute for Research on Alzheimer's Disease and \\ the Aging Brain, College of Physicians and Surgeons and ${ }^{\mathrm{d}}$ Department of Epidemiology, Mailman School of Public \\ Health, Columbia University, New York, N.Y., USA
}

\section{Key Words}

Essential tremor · Clinical features · Epidemiology · Biology · Pathophysiology · Duration · Age · Head tremor

\begin{abstract}
Background: Essential tremor (ET) is a chronic, progressive neurological disease. Head (neck) tremor may eventually develop in as many as $30-60 \%$ of patients, yet it is unclear why. Is its appearance merely a function of advancing disease duration? Alternatively, is patient age a primary factor? The latter would argue for the presence of a biological clock that is important for the expression of this clinical feature of ET. Methods: A total of 363 ET patients were enrolled in a cross-sectional, clinical-epidemiological study. Each ET patient underwent a 20-min videotaped neurological examination which included an assessment of the presence/absence of head tremor. Results: Head tremor was present on examination in 140 (38.6\%) patients. Young patients, even with longer-duration tremor, rarely had head tremor: $2 / 27$ (7.4\%) patients $<40$ years old with tremor duration $\geq 10$ years had head tremor versus $121 / 283(42.8 \%$ ) older patients ( $>60$ years old) with tremor duration $\geq 10$ years $(p<0.001)$. In a multivariate logistic regression analysis, while head tremor was associated with age $(p<0.001)$ it was not independently associated with tremor duration $(p=0.26)$; interestingly, it was associated with gender in that model $(p<0.001)$. With the exception of 1 patient, head tremor did not begin before
\end{abstract}

the age of 36. Conclusions: Data suggest that the appearance of head tremor in ET depends on a biological factor that is intrinsic to the patient (i.e. age) and is not a clear consequence of advancing disease duration.

Copyright $\odot 2013$ S. Karger AG, Basel

\section{Introduction}

While some diseases are the result of a single, identifiable environmental exposure, many are associated with enhanced genetic susceptibility or both genetic and environmental factors. Diseases tend to express themselves at certain points in the life cycle. They also tend to follow a patterned expression, with what is often a predictable unfolding of clinical features. What turns a disease on and then further drives its expression at certain points? This is frequently unknown but is often considered complex and multifactorial. On a very basic level, it can even be difficult to disentangle the influences of disease duration (a factor that is intrinsic to the disease) versus age (a biological factor that is intrinsic to the patient) on clinical expression.

Head (neck) tremor is a major feature of essential tremor (ET), generally developing some time after the clinical expression of upper limb tremor $[1,2]$. Head tremor does not occur in all ET patients; it only occurs in some, with prevalence estimates ranging from 30 to $60 \%$ $[1,3]$. For patients, head tremor is particularly problem-

Dr. Elan Louis, MD, MSc

Unit 198, Neurological Institute

710 West 168th Street

New York, NY 10032 (USA)

E-Mail EDL2@columbia.edu 
atic because it is easily seen and difficult to conceal, often embarrassing, and may be more resistant to treatment than upper limb tremor [4-6]. Curiously, studies of pediatric ET indicate that head tremor is a very uncommon occurrence in children with this disease [7].

To our knowledge, prior investigators have not tried to unravel whether tremor duration, age, or both are driving the expression of this common yet inconstant feature of ET. If it were age, this would argue for the presence of a 'biological clock' that drives clinical expression. It brings to the fore the interesting notion that factors that are intrinsic to the patient could be interacting with their disease to modify its clinical expression.

The current analyses utilized cross-sectional data on more than 350 ET patients of various ages and durations to try to untangle the effects of age from duration on the expression of head tremor. Our aim is that the presented data will be of value in clinical settings, which are often enhanced by the availability of empirical knowledge and an understanding of clinical patterns.

\section{Methods}

The current analyses were secondary analyses in a larger study. ET patients, aged 18 years and older, were enrolled in a clinicalepidemiological study that assessed environmental risk factors for ET $[8,9]$. Recruitment began in 2000 and ended in 2009. By design, they were identified from several sources; the major ones were a computerized billing database of patients at the Center for Parkinson's Disease and Other Movement Disorders at Columbia University Medical Center (CUMC), and the International Essential Tremor Foundation, whose members lived in the New York Metropolitan area and were mailed advertisements. All patients had received a diagnosis of ET from their treating neurologist and lived within a 2-hour driving distance of CUMC. Diagnoses were confirmed based on chart review and videotaped neurological examination by a senior movement disorder neurologist (E.D.L.) who applied published diagnostic criteria for ET (moderate or greater amplitude kinetic tremor during three activities or a head tremor in the absence of Parkinson's disease, dystonia or another known cause of tremor, e.g. thyroid disease or drug-induced tremor). ET diagnoses were also consistent with the consensus statement of the Movement Disorders Society regarding classic ET [10]. A Telephone Interview for Cognitive Status [11] was administered and cases were excluded if they had evidence of cognitive impairment (score $<30$ out of 41 ).

The study was cross-sectional; once enrolled, each patient had a single in-person evaluation. Each patient signed a written informed consent approved by the CUMC Institutional Review Board and was evaluated in person by a trained tester. Demographic and clinical information, including family history information, was collected using semistructured questionnaires. Age of tremor onset was by self-report; it was the age at which the individual first noted tremor. Generally, this was upper limb tremor; age of head tremor onset was not recalled by most patients. Prior studies have indicated that age of onset of limb tremor is reliably reported by ET patients [12]. Tremor duration was the difference between current age and age of tremor onset.

Each ET patient underwent a 20-min videotaped neurological examination, which included a detailed assessment of postural tremor and five tests of kinetic tremor, e.g. drinking from a cup and drawing spirals $[8,9]$. E.D.L. reviewed all videotaped examinations, and the severity of postural and kinetic arm tremors were rated $(0-3)$, resulting in a total tremor score (range $=0-36$, max.), which is a measure of the severity of action tremor. Ratings of 2 indicated moderate amplitude tremor; ratings of 3 were applied when the tremor was severe, (e.g. spilling when handling liquids or marked tremor while writing (see example of writing in fig. 5 of Louis et al. [13]). Neck (head) tremor was assessed at multiple points during the videotape, including while seated comfortably and facing the camera, and was noted as present or absent. Head tremor in ET was distinguished from dystonic tremor by the absence of twisting or tilting movements of the neck, jerk-like or sustained neck deviation, or hypertrophy of neck muscles. None of the ET cases had dystonia in their limbs.

Statistical analyses were carried out using SPSS version 19 (SPSS, Chicago, Ill., USA). Stratified data were first assessed and presented (using simple cross-tabulations) to provide a basic sense of the relationship between presence/absence of head tremor and each of the time variables (age and duration). Patients were thus stratified into 7 age groups and 6 tremor duration groups. With the exception of the youngest and oldest age categories, these categories were by 10 -year intervals. $\chi^{2}$ tests were used to assess differences in proportions. To increase the sample size within individual strata, in additional analyses, the time variables were divided into quartiles rather than 10-year age intervals. Neither age nor duration was normally distributed (Kolmogorov-Smirnov p values $<0.001$ ); hence, a nonparametric test (Spearman's $\rho$ ) was used to assess their correlation with one another. Bivariate as well as multivariate logistic regression analyses were used in which head tremor was the dependent variable; the main independent variables of interest were age, age stratum, tremor duration, tremor duration stratum, gender, and family history of tremor in various models. In logistic regression models we also assessed the odds of head tremor as a function of tremor duration in each of 4 age quartiles and, conversely, we assessed the odds of head tremor as a function of age in each of 4 tremor duration quartiles. The use of quartiles rather than smaller categories (e.g. quintiles) provided adequate numbers (approx. 90 cases) in each stratum.

\section{Results}

There were 363 ET patients (table 1). The mean age was $67.4 \pm 15.3$ years $($ range $=18-95)$, and the mean tremor duration was $23.0 \pm 18.6$ years (range $=1-81$; table 1 ). Head tremor was present on examination in 140 patients (38.6\%); tremor severity in the arms ranged from very mild to severe (total tremor score $=18.9 \pm 7.2$ ).

Patients were stratified into 7 age groups and 6 tremor duration groups (tables 1,2). The proportion of patients with head tremor clearly increased in successive age stra- 
Table 1. Demographic and clinical characteristics of 363 ET patients

\begin{tabular}{|c|c|}
\hline Age, years & $67.4 \pm 15.3(18-95)$ \\
\hline \multicolumn{2}{|l|}{ Age group } \\
\hline$\leq 30$ years $^{\mathrm{a}}$ & $11(3.0)$ \\
\hline $31-40$ years & $22(6.1)$ \\
\hline $41-50$ years & $18(5.0)$ \\
\hline $51-60$ years & $29(8.0)$ \\
\hline $61-70$ years & $95(26.2)$ \\
\hline $71-80$ years & $129(35.5)$ \\
\hline$\geq 81$ years & $59(16.3)$ \\
\hline Female gender & $184(50.7)$ \\
\hline White race & $341(93.9)$ \\
\hline Education, years & $15.1 \pm 3.8$ \\
\hline Tremor duration, years & $23.0 \pm 18.6(1-81)$ \\
\hline \multicolumn{2}{|l|}{ Tremor duration group } \\
\hline $0-10$ years & $132(36.4)$ \\
\hline $11-20$ years & $72(19.8)$ \\
\hline $21-30$ years & $58(16.0)$ \\
\hline $31-40$ years & $30(8.3)$ \\
\hline $41-50$ years & $29(8.0)$ \\
\hline$\geq 50$ years & $42(11.6)$ \\
\hline Age of tremor onset, years & $44.6 \pm 22.6$ \\
\hline Currently takes medication to treat ET & $196(54.0)$ \\
\hline Family history of tremor ${ }^{b}$ & $225(62.0)$ \\
\hline Total tremor score on neurological & $18.9 \pm 7.2$ \\
\hline \multicolumn{2}{|l|}{$\begin{array}{l}\text { Head (neck) tremor on neurological } \\
\text { examination }\end{array}$} \\
\hline $\begin{array}{l}\text { All values are presented as mean } \pm \\
\text { range) or number (with percent), unles } \\
\text { a All participants were } \geq 18 \text { years of } \\
\text { gree relative reported to have tremor. }\end{array}$ & $\begin{array}{l}\text { dard deviation (with } \\
\text { erwise specified. } \\
\text { First- or second-de }\end{array}$ \\
\hline
\end{tabular}

ta $(\mathrm{p}<0.001$; table 2); there was a marginal increase in the proportion of patients with head tremor in the higher tremor duration strata $(\mathrm{p}=0.054$; table 2$)$. We also assessed the odds of head tremor in each of 4 age quartiles; in a series of 4 bivariate logistic regression analyses, there was no association between tremor duration (independent variable) and odds of head tremor (dependent variable; all $p$ values $>0.05$; table 3 ). By contrast, we assessed the odds of head tremor in each of 4 tremor duration quartiles; there was an association between age (independent variable) and odds of head tremor (dependent variable) in 3 of the 4 quartiles ( 3 p values $\leq 0.01$; table 3 ).
Table 2. Head tremor by age stratum and tremor duration stratum Number with head tremor p value (entire sample, $\mathrm{n}=363$ )

$\begin{array}{lr}\begin{array}{l}\text { Age group } \\ \leq 29 \text { years } \\ \text { a }\end{array} & 1(9.1) \\ 30-39 \text { years } & 2(9.1) \\ 40-49 \text { years } & 5(33.3) \\ 50-59 \text { years } & 9(36.0) \\ 60-69 \text { years } & 25(28.4) \\ 70-79 \text { years } & 63(47.0) \\ \geq 80 \text { years } & 35(51.5)\end{array}$

Tremor duration group

$\begin{array}{ll}0-10 \text { years } & 47(35.6) \\ 11-20 \text { years } & 24(33.3) \\ 21-30 \text { years } & 22(37.9) \\ 31-40 \text { years } & 14(46.7) \\ 41-50 \text { years } & 11(37.9) \\ \geq 50 \text { years } & 22(52.4)\end{array}$

$0.054^{\mathrm{c}}$

All values are presented as number (with percent).

a All participants were $\geq 18$ years of age.

${ }^{b}$ In a logistic regression analysis in which head tremor was the dependent variable and age group was the independent variable, $\mathrm{OR}=1.5(95 \% \mathrm{CI}=1.23-1.73, \mathrm{p}<0.001)$.

${ }^{\mathrm{c}}$ In a logistic regression analysis in which head tremor was the dependent variable and tremor duration group was the independent variable, $\mathrm{OR}=1.1(95 \% \mathrm{CI}=0.998-1.27, \mathrm{p}=0.054)$.

Table 3. Odds of head tremor as a function of tremor duration or age: quartile analysis

OR $\quad 95 \% \mathrm{CI} \quad \mathrm{p}$ value

Odds of head tremor as a function of tremor duration ${ }^{\mathrm{a}}$

Age quartile

$\begin{array}{llrl}\text { Quartile 1 ( } \leq 62 \text { years) } & 1.01 & 0.98-1.05 & 0.45 \\ \text { Quartile 2 (63-70 years) } & 0.99 & 0.97-1.02 & 0.52 \\ \text { Quartile 3 (71-77 years) } & 1.02 & 0.998-1.04 & 0.07 \\ \text { Quartile 4 ( } \geq 78 \text { years) } & 1.01 & 0.99-1.03 & 0.20\end{array}$

Odds of head tremor as a function of age $\mathrm{b}^{\mathrm{b}}$

Tremor duration quartile

$\begin{array}{llll}\text { Quartile } 1 \text { ( } \leq 7 \text { years }) & 1.01 & 0.98-1.04 & 0.63 \\ \text { Quartile 2 (8-17 years) } & 1.04 & 1.01-1.08 & 0.01 \\ \text { Quartile 3 (18-33 years) } & 1.04 & 1.01-1.07 & 0.008 \\ \text { Quartile 4 ( } \geq 34 \text { years) } & 1.07 & 1.02-1.12 & 0.01\end{array}$

${ }^{a}$ Each OR represents the result of a single bivariate logistic regression analysis in 1 age quartile. Dependent variable $=$ head tremor and independent variable $=$ tremor duration in years.

${ }^{b}$ Each OR represents the result of a single bivariate logistic regression analysis in 1 tremor duration quartile. Dependent variable $=$ head tremor and independent variable $=$ age in years. 
Fig. 1. ET patients by age and tremor duration. Patients with head tremor are indicated by blue circles (color refers to online version only); patients without head tremor are indicated by red squares.
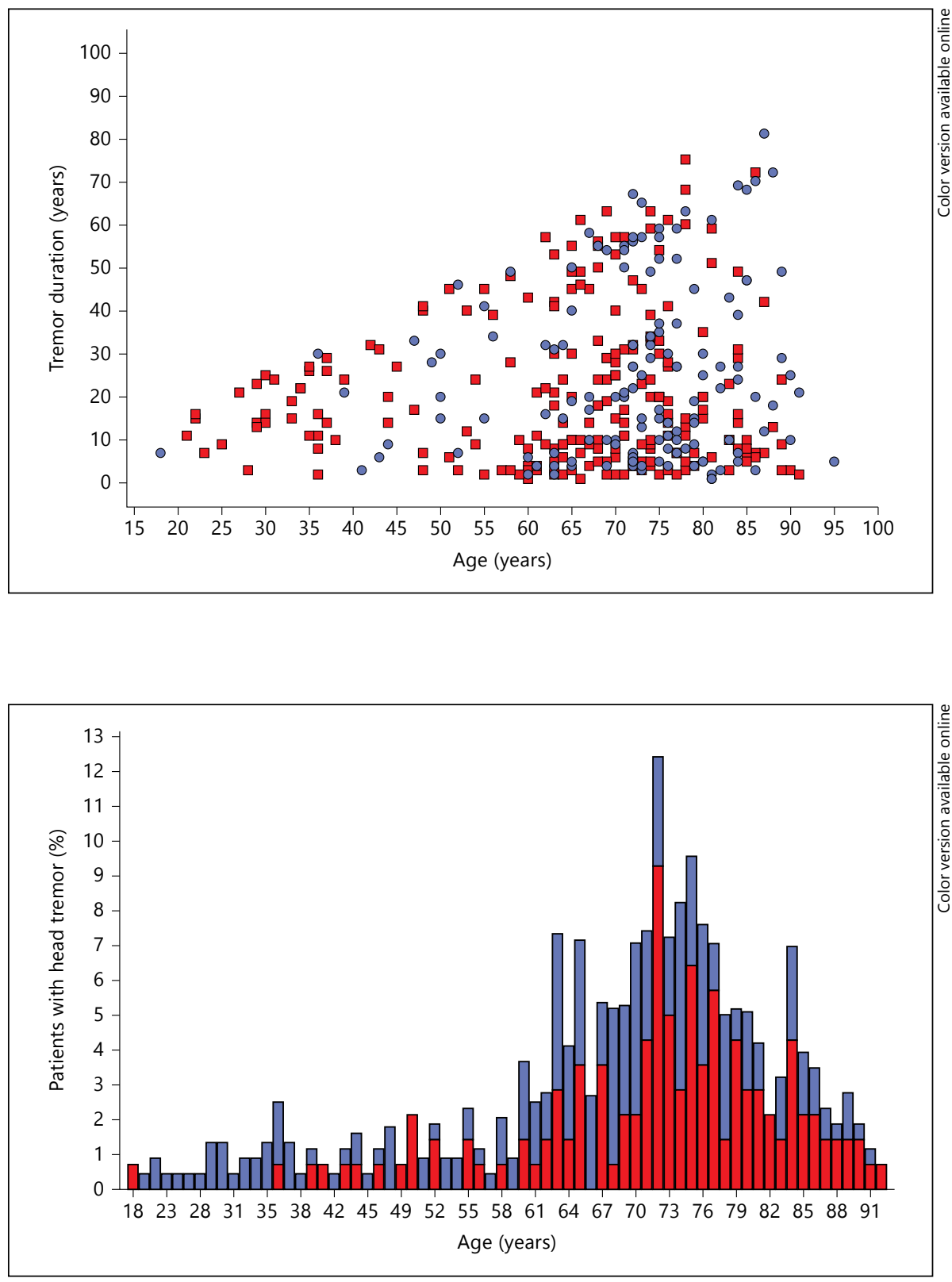

Fig. 2. Percentage of ET patients with head tremor (red bar; color refers to online version only) and without head tremor (blue bar) at each age. The percentage is the percentage of all 363 ET patients.
Age and tremor duration were not correlated with one another (Spearman's $\rho=0.06, p=0.29$ ).

In a bivariate logistic regression analysis, head tremor (dependent variable) was associated with age in years (odds ratio, $\mathrm{OR}=1.04,95 \%$ confidence interval, $\mathrm{CI}=$ $1.02-1.05, \mathrm{p}<0.001)$. In a second bivariate logistic regression analysis, head tremor was weakly associated with tremor duration in years $(\mathrm{OR}=1.01,95 \% \mathrm{CI}=1.002$ $1.024, \mathrm{p}=0.03)$. In a multivariate logistic regression analysis, head tremor was associated with age in years $(\mathrm{OR}=$ $1.04,95 \% \mathrm{CI}=1.02-1.05, \mathrm{p}<0.001)$ and marginally as- sociated with duration in years $(\mathrm{OR}=1.01,95 \% \mathrm{CI}=$ $0.999-1.022, \mathrm{p}=0.08)$. In a second multivariate logistic regression analysis, head tremor was associated with age in years $(\mathrm{OR}=1.04,95 \% \mathrm{CI}=1.02-1.06, \mathrm{p}<0.001)$ and female gender $(\mathrm{OR}=3.7,95 \% \mathrm{CI}=2.33-5.94, \mathrm{p}<0.001)$, but not tremor duration $(\mathrm{OR}=1.007,95 \% \mathrm{CI}=0.995-$ $1.02, \mathrm{p}=0.26)$ or family history of tremor $(\mathrm{OR}=1.27,95 \%$ $\mathrm{CI}=0.77-1.20, \mathrm{p}=0.35$ ).

With the exception of 1 female patient, head tremor did not begin before the age of 36 (fig. 1,2). Of the patients who were $<40$ years of age, 27 had a tremor dura- 
tion of $\geq 10$ years; only $2 / 27$ (7.4\%) had head tremor. Hence, even young patients with longer-duration ET rarely had head tremor. By comparison, of the 283 patients who were $>60$ years of age and had tremor for $\geq 10$ years, $121 / 283(42.8 \%)$ had head tremor $\left(\chi^{2}=12.87\right.$, $\mathrm{p}<0.001)$. Whereas head tremor was rare in young individuals, it was present in more than one third (39/114, $34.2 \%)$ of persons in the shortest tremor duration group (0-9 years). There were 52 patients with tremor $<5$ years in duration (age $=67.9 \pm 12.9$ years, range $=29-91$ ); one third of these $(17 / 52,32.7 \%)$ had head tremor.

The youngest male with head tremor was 47 years old; the youngest female was 18 . Among those $<50$ years, $2 / 24$ (8.3\%) males (aged 47 and 49 years) versus $6 / 24$ (25.0\%) females (aged 18, 36, 39, 41, 43 and 44 years) had head tremor (Fisher's $\mathrm{p}=0.24$ ).

The total tremor score was divided into quartiles $(\leq 13$, $14-18,19-23, \geq 24)$ and there was a tendency for the proportion of patients with head tremor to increase from lowest to highest quartile: $30 / 79(38.0 \%)$ in lowest quartile, 23/99 (23.2\%) in quartile 2, 41/94 (43.6\%) in quartile 3 , and $44 / 85(51.8 \%)$ in the highest quartile $\left(\chi^{2}=17.08\right.$, $\mathrm{p}=0.001)$.

\section{Discussion}

It is difficult to disentangle the influences of age (a biological factor that is intrinsic to the patient) versus duration (a factor that is intrinsic to the disease) on disease expression. Clinicians are often faced with ET patients among whom both age and duration are increasing at the same time, making it difficult to separate the effects of one from the other. Also, the wide range of ages and durations among the typical clinician's patient sample can make it difficult to piece together any type of overall pattern, and it is difficult to account for the independent effects of age and duration without formal statistical comparisons. The ability to study a full range of patients, including younger patients with longer tremor duration and older patients with shorter tremor duration, allows one to more decisively separate these two factors. Our data, from a large sample of ET patients, indicate that the appearance of head tremor in ET is rare before the age of 40 and that its presence was determined more clearly by the age of the patient rather than the duration of their tremor.

A biological clock is an inherent periodicity in the physiological processes of a living organism that is not dependent on the periodicity of external factors. The appearance of head tremor in ET seems to depend on a bio- logical factor that is intrinsic to the patient (age or aging) and is not the independent consequence of advancing disease duration. Studies of pediatric ET cases have indicated that head tremor among children with ET is very rare. Indeed, a study of 95 pediatric ET cases from three centers indicated that only 3 (3.2\%) had head tremor [14]. A study in Singapore of 17 young-onset ET cases found that none $(0.0 \%)$ had head tremor [15]. Another study of 39 ET patients (age range $=3.3-64.4$ ) with young-onset ET indicated that 7 (17.9\%) had head tremor; however, 11 of these $39 \mathrm{ET}$ patients also had dystonia [16].

Gender is another intrinsic factor that has been linked with head tremor in ET. Both in this sample and in prior studies, head tremor is more common in women with ET than in men $[1,17]$. This brings to the fore the notion that factors that are intrinsic to the ET patient (age and gender) could be interacting with their disease to modify its clinical expression. The reason why female gender seems to predispose ET patients to develop head tremor is not known.

The traditional explanation for the presence of head tremor in ET is that head tremor may require more extensive brain involvement, which occurs only later in the disease [15]. We have demonstrated that although on the surface head tremor seems to be related to disease duration in ET, when one properly accounts for the effects of age, using both stratified and adjusted analyses, the occurrence of head tremor in ET is a function of age rather than disease duration.

This study has limitations. First, the data were crosssectional rather than longitudinal; hence the tremor duration groups represent different individuals rather than a cohort of the same individuals who were sampled over time. Second, while age was known precisely, the precise age of onset in ET may be more difficult to establish. While this differential precision could explain some of our findings, many of our analyses involved 10-year duration intervals in which 1-year precision was not necessary. Our prior study has shown that two thirds of ET cases are able to reliably report age of their onset to within 4 years [18]. Furthermore, the primary error in misremembering the duration of tremor is likely to have been in the form of 'telescoping' (i.e. errors primarily in the direction of remembering an event as having occurred more recently than it did). This is most likely to have affected individuals with durations of up to 30 years rather than extremely long durations. Even if an individual with 15 years of tremor reported ' 5 ' years of tremor or an individual with 30 years of tremor reported only ' 20 ' years of tremor, this would not have affected our results, as the 
proportion with head tremor in these duration groups was strikingly similar (table 2). Third, this was not a population-based study; the associations we report need to be reproduced in a population-based sample of ET cases.

The study also had a number of strengths. First, the large sample size of more than $350 \mathrm{ET}$ patients provided enough power for a broad range of analyses. Second, the sample included patients with a wide range in disease duration; in some instances this was $>80$ years and in others it was as short as 1 year. Similarly, age was broadly represented in this sample. Third, the evaluation was applied to all patients in a uniform manner and included a range of assessment tools including neurological examination items.

These and other published data suggest that the appearance of head tremor in ET depends on a biological factor that is intrinsic to the patient (i.e. age). Our aim is that the presented data will be of value in clinical settings, which are often enhanced by the availability of empirical knowledge and an understanding of clinical patterns.

\section{Acknowledgements}

This study was funded by National Institutes of Health Grant R01 NS039422 to the author.

\section{Disclosure Statement}

The author declares that there are no conflicts of interest and no competing financial interests.

\section{References}

$>1$ Louis ED, Ford B, Frucht S: Factors associated with increased risk of head tremor in essential tremor: a community-based study in northern Manhattan. Mov Disord 2003;18:432-436.

$>2$ Benito-Leon J, Louis ED: Clinical update: diagnosis and treatment of essential tremor. Lancet 2007;369:1152-1154.

-3 Louis ED, Ford B, Barnes LF: Clinical subtypes of essential tremor. Arch Neurol 2000; 57:1194-1198.

4 Putzke JD, Uitti RJ, Obwegeser AA, Wszolek ZK, Wharen RE: Bilateral thalamic deep brain stimulation: midline tremor control. J Neurol Neurosurg Psychiatry 2005;76:684-690.

$>5$ Song IU, Kim JS, Lee SB, Ryu SY, An JY, Kim HT, Kim YI, Lee KS: Effects of zonisamide on isolated head tremor. Eur J Neurol 2008;15: 1212-1215.

6 Zesiewicz TA, Elble R, Louis ED, Hauser RA, Sullivan KL, Dewey RB Jr, Ondo WG, Gronseth GS, Weiner WJ: Practice parameter: therapies for essential tremor: report of the Quality Standards Subcommittee of the American Academy of Neurology. Neurology 2005;64:2008-2020.
7 Louis ED, Dure LS 4th, Pullman S: Essential tremor in childhood: a series of nineteen cases. Mov Disord 2001;16:921-923.

$>8$ Louis ED, Jiang W, Pellegrino KM, Rios E, Factor-Litvak P, Henchcliffe C, Zheng W: Elevated blood harmane (1-methyl-9H-pyrido[3,4b]indole) concentrations in essential tremor. Neurotoxicology 2008;29:294-300.

$\checkmark 9$ Louis ED, Huey ED, Gerbin M, Viner AS: Depressive traits in essential tremor: impact on disability, quality of life, and medication adherence. Eur J Neurol 2012;19:1349-1354.

10 Deuschl G, Bain P, Brin M: Consensus statement of the Movement Disorder Society on Tremor. Ad Hoc Scientific Committee. Mov Disord 1998;13(suppl 3):2-23.

11 Brandt J, Folstein M: The Telephone Interview for Cognitive Status. Neuropsychiatry Neuropsychol Behav Neurol 1988;1:111-117.

12 Louis ED, Schonberger RB, Parides M, Ford B, Barnes LF: Test-retest reliability of patient information on age of onset in essential tremor. Mov Disord 2000;15:738-741.
13 Louis ED, Zhao Q, Meng H, Ding D: Screening for action tremor in epidemiological field surveys: assessing the reliability of a semiquantitative, visual, template-based scale for rating hand-drawn spirals. Tremor Other Hyperkinet Mov (NY) 2012;2:pii.

14 Louis ED, Fernandez-Alvarez E, Dure LS 4th, Frucht S, Ford B: Association between male gender and pediatric essential tremor. Mov Disord 2005;20:904-906.

15 Tan EK, Lum SY, Prakash KM: Clinical features of childhood onset essential tremor. Eur J Neurol 2006;13:1302-1305.

16 Jankovic J, Madisetty J, Vuong KD: Essential tremor among children. Pediatrics 2004;114: 1203-1205.

17 Hubble JP, Busenbark KL, Pahwa R, Lyons K, Koller WC: Clinical expression of essential tremor: effects of gender and age. Mov Disord 1997;12:969-972.

18 Louis ED: Age of onset: can we rely on essential tremor patients to report this? Data from a prospective, longitudinal study. Neuroepidemiology 2013;40:93-98. 